\title{
An improvement in ABIC-minimizing deconvolution for continuously measured magnetic remanence data
}

\author{
Hirokuni Oda ${ }^{1}$ and Hidetoshi Shibuya ${ }^{2}$ \\ ${ }^{1}$ Marine Geology Department, Geological Survey of Japan, Tsukuba 305, Japan \\ ${ }^{2}$ Department of Earth Sciences, Kumamoto University, Kumamoto 860, Japan \\ (Received May 15, 1996; Revised September 8, 1997; Accepted September 27, 1997)
}

\begin{abstract}
Deconvolution of magnetic remanence which has been continuously measured along a long-core sample is improved by eliminating the disturbance of severe intensity variation confined to a narrow layer. The deconvolution is constrained by the smoothness of the magnetization measured by the $L_{2}$-norm of second order difference. The previous model uses a single smoothness parameter for the entire sample and the optimum smoothness is obtained by minimizing ABIC (Akaike's Bayesian Information Criterion), while an additional parameter is introduced in the modified scheme for a layer where intensity fluctuation is strong. The modified deconvolution scheme was applied to 5-mm interval magnetic remanence data for two sections of ODP Hole 767B. We assumed another smoothness for the uppermost $1.5 \mathrm{~cm}$ of Section 767B-6H1 where the coring disturbance is severe. For Section 767B-6H2, the Australasian microtektite layer $(108-113 \mathrm{~cm})$, where the magnetization intensity shows a strong peak, is expressed by a different smoothness parameter from other part. In both cases the minimum ABIC was lowered and the calculated error was reduced, demonstrating the improvement of the model by incorporating additional information other than magnetic remanence data in the prior distribution of the data.
\end{abstract}

\section{Introduction}

Magnetic data, which are measured by a pass-through magnetometer with a broad response function, have to be deconvolved to reveal detailed variations. As the deconvolution operation tends to be ill-natured, some constraints are needed to stabilize the results. Deconvolution of magnetic remanence data from long-core samples was first attempted by Dodson et al. (1974) with the help of a lowpass-filter in the frequency space. However, their method is not objective because they determined the optimum filter shape visually on display. Constable and Parker (1991) developed a smoothness penalized deconvolution with cubic spline expressions in which the optimum smoothness is determined by adjusting the misfit between the data and the model to the measured noise level. Oda and Shibuya (1994) pointed out that the deconvolution scheme by Constable and Parker (1991) leads to an unstable solution when the noise level is underestimated.

Oda and Shibuya (1994) introduced a Bayesian deconvolution method in the form of smoothness penalized least squares fitting. The optimum smoothness parameter is determined by minimizing Akaike's Bayesian Information Criterion (ABIC) proposed by Akaike (1980) and the appropriate noise level is estimated at the same time. Oda and Shibuya (1996) further developed the ABIC-minimizing deconvolution for ODP pass-through paleomagnetic data in the three dimensional form incorporating cross terms. The ABIC-minimizing deconvolution was applied to the pass-

Copy right $\odot$ The Society of Geomagnetism and Earth, Planetary and Space Sciences (SGEPSS); The Seismological Society of Japan; The Volcanological Society of Japan; The Geodetic Society of Japan; The Japanese Society for Planetary Sciences. through data of the Brunhes/Matuyama polarity transition from three holes of ODP Leg 124 (Oda, 1995). For Holes $769 \mathrm{~A}$ and $769 \mathrm{~B}$, the deconvolution was quite successful and the results are consistent with each other, whereas the estimated magnetization was noisy for Hole 767B after the deconvolution. The large error is suspected to be due to thin layers that have several times stronger magnetizations than in other parts. Possible cause is disturbance at the core top or sudden lithological transition such as volcanic ash layers. Thus, we developed a modified deconvolution scheme that can eliminate the disturbance of a sudden intensity change by the introduction of two smoothness parameters. The new scheme was applied to the pass-through paleomagnetic data from ODP Hole 767B.

\section{Formulation}

The basic formulation follows Oda and Shibuya (1996). The continuous measurement of the magnetic remanence of a long-core sample is expressed by unit slices of homogeneous magnetization, in the vector-matrix representation:

$$
\boldsymbol{d}=\boldsymbol{R} \boldsymbol{m}+\boldsymbol{e}
$$

where $\boldsymbol{d}$ denotes the magnetic moment which is the output of the sensor, $\boldsymbol{m}$ the magnetization of the sample, $\boldsymbol{R}$ the sensor response matrix, and $\boldsymbol{e}$ the noise in measurements. The configuration of ODP's magnetometer using half-column samples with flat surface facing up results in cross-terms between $x$ (downward) and $z$ (down-core) axes (Oda and Shibuya, 1996). Both the vectors and the response matrix can be expressed by $x, y$, and $z$ components as 


$$
\left[\begin{array}{l}
\boldsymbol{d}_{x} \\
\boldsymbol{d}_{y} \\
\boldsymbol{d}_{z}
\end{array}\right]=\left[\begin{array}{ccc}
\boldsymbol{R}_{x x} & 0 & \boldsymbol{R}_{z x} \\
0 & \boldsymbol{R}_{y y} & 0 \\
\boldsymbol{R}_{x z} & 0 & \boldsymbol{R}_{z z}
\end{array}\right]\left[\begin{array}{l}
\boldsymbol{m}_{x} \\
\boldsymbol{m}_{y} \\
\boldsymbol{m}_{z}
\end{array}\right]+\left[\begin{array}{c}
\boldsymbol{e}_{x} \\
\boldsymbol{e}_{y} \\
\boldsymbol{e}_{z}
\end{array}\right] .
$$

The main source of the noise is considered to be mispositioning in the measurement, lateral heterogeneity of the magnetization (distortion by drilling), and discrepancy of the estimated sensor response from the true one (Oda and Shibuya, 1996). However, the noise in the three components are assumed to be independent and subjected to the Gaussian noise in the first order approximation as a mixture of various noise sources, resulting in easy calculation. The mean of the noise is assumed to be zero and its variance is $\tau$. As a result, the probability distribution of the whole-core data is given by

$$
L(d \mid \boldsymbol{m}, \tau)=\left(\frac{1}{2 \pi \tau}\right)^{\frac{3 N}{2}} \exp \left\{-\frac{1}{2 \tau}\|\boldsymbol{d}-\boldsymbol{R} \boldsymbol{m}\|^{2}\right\}
$$

where $N$ is the number of measured points.

\subsection{Release from end disturbance}

The deconvolution is stabilized by introducing smoothness constraints by $L_{2}$-norm of second order difference for the magnetization. In terms of the Bayesian statistics, this stabilization is accomplished by a prior distribution based on the knowledge that the magnetization changes smoothly. Oda and Shibuya (1996) used a smoothness parameter $u$ for the whole core sample to suppress the fluctuations, assuming that the smoothness of the magnetization does not vary throughout the section. In order to introduce a knowledge that the end of the core is disturbed and the magnetization of this part fluctuates strongly (e.g. mechanically and magnetically disturbed top core of Section 767B-6H1), the prior distribution was changed to include another smoothness parameter for this portion. The parameter $u$ is adopted for the normal undisturbed part of the core which was used by Oda and Shibuya (1996) and the other is a newly defined parameter $v$ for the disturbed top part from point 1 to point $K$. Using these two hyperparameters, the smoothness of the magnetization is expressed as

$$
\Delta^{2}=\Delta_{x}^{2}+\Delta_{y}^{2}+\Delta_{z}^{2}
$$

where

$$
\begin{aligned}
\Delta_{\xi}^{2} & =v^{2}\left(\alpha_{\xi 1}^{2} m_{\xi 1}^{2}+\beta_{\xi 1}^{2}\left|m_{\xi 2}-m_{\xi 1}\right|^{2}+\sum_{i=3}^{K}\left|m_{\xi i}-2 m_{\xi i-1}+m_{\xi i-2}\right|^{2}\right) \\
& +u^{2}\left(\alpha_{\xi_{2}}^{2} m_{\xi K+1}^{2}+\beta_{\xi 2}^{2}\left|m_{\xi K+2}-m_{\xi K+1}\right|^{2}+\sum_{i=K+3}^{M}\left|m_{\xi_{i}}-2 m_{\xi i-1}+m_{\xi i-2}\right|^{2}\right) .
\end{aligned}
$$

Here $M$ is the number of uniformly magnetized unit slices, $\xi=(x, y, z)$, and $\alpha_{\xi 1}, \beta_{\xi 1}, \alpha_{\xi 2}$, and $\beta_{\xi_{2}}$ are properly chosen parameters that can be optimized as part of a least squares fitting of Eq. (10) described below (detailed formulation follows Oda and Shibuya, 1996). This incorporates change in the smoothness of the magnetization such as sudden lithological change, disturbance by coring, and rust contamination. Equation (4) can be expressed simply by the matrix as

$$
\left\|\boldsymbol{D}_{u, v} \boldsymbol{m}\right\|^{2}
$$

where

$$
\boldsymbol{D}_{u, v}=\left[\begin{array}{ccc}
\boldsymbol{D}_{1, u, v} & 0 & 0 \\
0 & \boldsymbol{D}_{1, u, v} & 0 \\
0 & 0 & \boldsymbol{D}_{1, u, v}
\end{array}\right] \text {. }
$$

Here $\boldsymbol{D}_{1, u, v}$ are defined by the following as

$$
\boldsymbol{D}_{1, u, v}=\left[\begin{array}{cc}
v \boldsymbol{D}_{2, K} & 0 \\
0 & u \boldsymbol{D}_{2, M-K}
\end{array}\right]
$$

where $\boldsymbol{D}_{2, p}$ is given by

$$
\boldsymbol{D}_{2, p}=\left(\begin{array}{cccc}
\alpha & & & \\
-\beta & \beta & & 0 \\
1 & -2 & 1 & \\
& \ddots & \ddots & \ddots \\
0 & 1 & -2 & 1
\end{array}\right)^{\cdots 1}
$$

Here $\alpha$ and $\beta$ are the parameters properly chosen for optimization as part of a least squares fitting described below (Eq. (10)). Using the expression above, the prior distribution of magnetization is written as

$$
\begin{aligned}
& p(\boldsymbol{m} \mid \tau, u, v) \\
& =a\left(\frac{1}{2 \pi \tau}\right)^{\frac{3 M}{2}}\left|\boldsymbol{D}_{u, v}{ }^{t} \boldsymbol{D}_{u, v}\right|^{\frac{1}{2}} \exp \left\{-\frac{1}{2 \tau}\left\|\boldsymbol{D}_{u, v} \boldsymbol{m}\right\|^{2}\right\}
\end{aligned}
$$

where $a$ is a normalization constant. According to the Bayes theorem, the posterior distribution is deduced to be

$$
p_{\text {post }}(\boldsymbol{m} \mid \boldsymbol{d}, \tau, u, v) \propto L(\boldsymbol{d} \mid \boldsymbol{m}, \tau) p(\boldsymbol{m} \mid \tau, u, v)
$$


which describes a probability distribution of the data under the condition of a prior distribution. Akaike (1980) proposed ABIC to obtain the optimum solution on the basis of the entropy maximization principle as follows.

$$
\begin{aligned}
\operatorname{ABIC}(\tau, u, v)= & -2 \log \int p_{\text {post }}(\boldsymbol{m} \mid \boldsymbol{d}, \tau, u, v) d \boldsymbol{m} \\
& +2 \text { (number of hyperparameters }) .
\end{aligned}
$$

By substitution of Eqs. (6) and (7) to Eq. (8) and successive transformations, we obtain

$$
\begin{aligned}
\operatorname{ABIC}(\tau, u, v)= & 3 N \log (2 \pi \tau)+\frac{S(u, v)}{\tau}-\log \left|\boldsymbol{D}_{u, v}{ }^{t} \boldsymbol{D}_{u, v}\right| \\
& +\log \left|\boldsymbol{R}^{t} \boldsymbol{R}+\boldsymbol{D}_{u, v}{ }^{t} \boldsymbol{D}_{u, v}\right|+6
\end{aligned}
$$

where

$$
S(u, v)=\left\|\boldsymbol{d}-\boldsymbol{R} \boldsymbol{m}_{0}\right\|^{2}+\left\|\boldsymbol{D}_{u, v} \boldsymbol{m}_{0}\right\|^{2}
$$

$\boldsymbol{m}_{0}$ is calculated from a least squares fitting for minimizing $S$. By differentiating Eq. (9) with respect to $\tau$, the value of $\tau$ which minimizes ABIC is determined as $\tau_{\min }=S / 3 N$ and the final form of $\mathrm{ABIC}$ is obtained as

$$
\begin{aligned}
\operatorname{ABIC}(u, v)= & 3 N \log \left(\frac{2 \pi S(u, v)}{3 N}\right)+3 N-\log \left|\boldsymbol{D}_{u, v}{ }^{t} \boldsymbol{D}_{u, v}\right| \\
& +\log \left|\boldsymbol{R}^{t} \boldsymbol{R}+\boldsymbol{D}_{u, v}{ }^{t} \boldsymbol{D}_{u, v}\right|+6 .
\end{aligned}
$$

The maximum likelihood of magnetization is obtained to be $\boldsymbol{m}_{0}$ together with the optimum values of $u$ and $v$ by minimization of ABIC.

\subsection{Release a thin layer}

For the case that a strongly magnetized layer such as volcanic ash layer is embedded in a normal layer, three
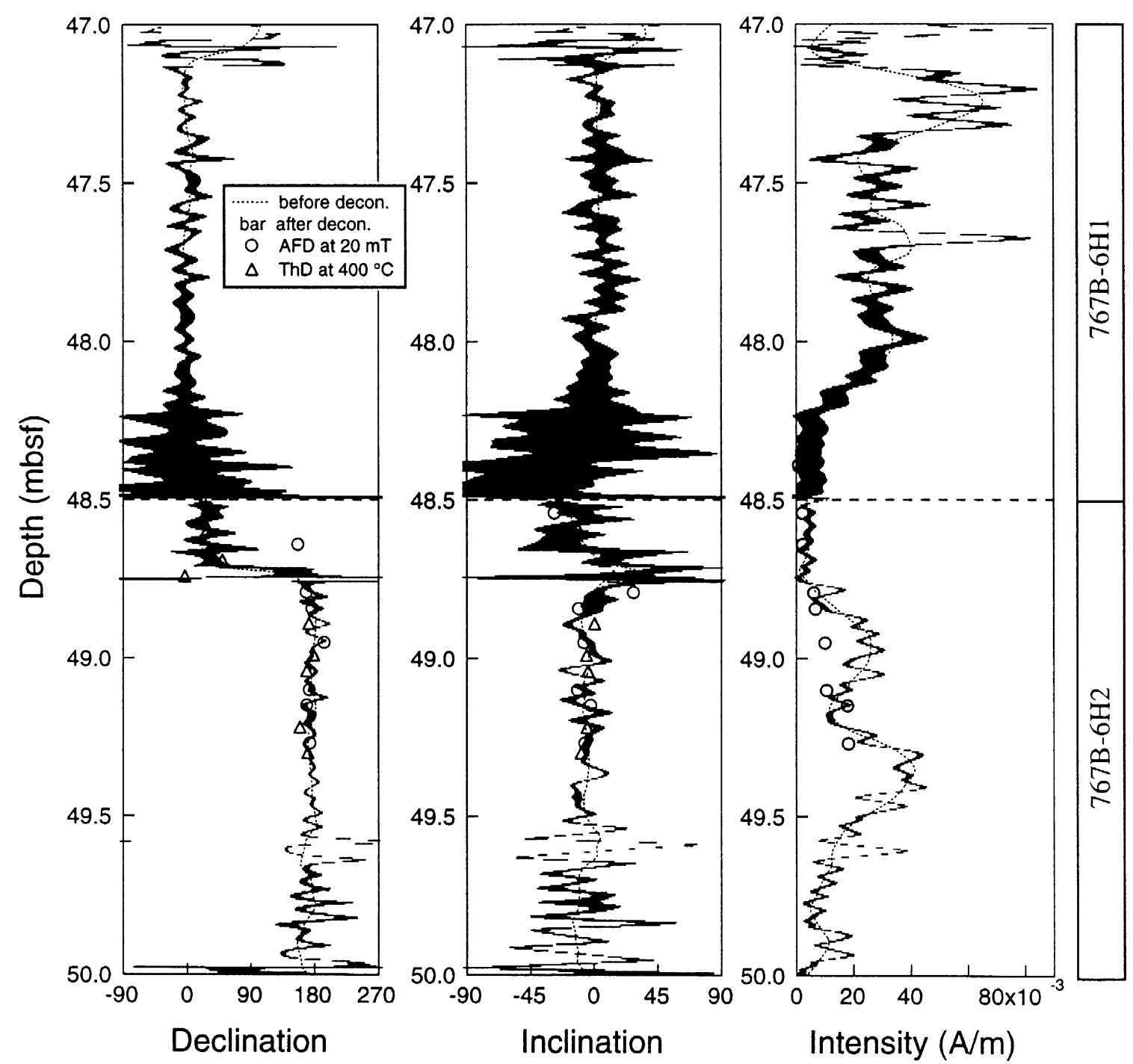

Fig. 1. The results of ABIC-minimizing deconvolution (Oda and Shibuya, 1996) for Sections 767B-6H1 (47.0-48.5 mbsf) and 767B-6H2 (48.5-50.0 $\mathrm{mbsf}$ ) plotted with depth for declination, inclination and intensity from left to right. Horizontal bars represent magnetization after the deconvolution. Each bar represents a 95\% confidence limit. Broken lines indicate magnetization before the deconvolution. Open circles and triangles represent paleomagnetic results of discrete cube samples after alternating field demagnetization at $20 \mathrm{mT}$ and thermal demagnetization at $400^{\circ} \mathrm{C}$, respectively. 
distinct parts are defined and different smoothness parameters are introduced. The layer of strong magnetizations begins at a data point $K_{1}$ and ends at a point $K_{2}$. In order to simplify the model the smoothness parameters of the two layers separated by the disturbed part are assumed to be the same. This can be expressed by using the new $\Delta_{\xi}$ instead of Eq. (4b) as

$$
\begin{aligned}
\Delta_{\xi}^{2} & =u^{2}\left(\alpha_{\xi 1}^{2} m_{\xi 1}^{2}+\beta_{\xi 1}^{2}\left|m_{\xi 2}-m_{\xi 1}\right|^{2}+\sum_{i=3}^{K_{1}}\left|m_{\xi_{i}}-2 m_{\xi i-1}+m_{\xi i-2}\right|^{2}\right) \\
& +v^{2}\left(\alpha_{\xi_{2}}^{2} m_{\xi K_{1}}^{2}+\beta_{\xi 2}^{2}\left|m_{\xi K_{1}+1}-m_{\xi K_{1}}\right|^{2}+\sum_{i=K_{1}+2}^{K_{2}}\left|m_{\xi_{i}}-2 m_{\xi_{i-1}}+m_{\xi i-2}\right|^{2}\right) \\
& +u^{2}\left(\alpha_{\xi 3}^{2} m_{\xi K_{2}+1}^{2}+\beta_{\xi 3}^{2}\left|m_{\xi K_{2}+2}-m_{\xi K_{2}+1}\right|^{2}+\sum_{i=K_{2}+3}^{M}\left|m_{\xi_{i}}-2 m_{\xi_{i-1}}+m_{\xi i-2}\right|^{2}\right)
\end{aligned}
$$

where $\alpha_{\xi 1}, \beta_{\xi 1}, \alpha_{\xi 2}, \beta_{\xi_{2}}, \alpha_{\xi 3}$, and $\beta_{\xi_{3}}$ are properly chosen parameters. This can be expressed by using the matrix modified from Eq. (5c):

$$
\boldsymbol{D}_{1, u, v}=\left[\begin{array}{ccc}
u \boldsymbol{D}_{2, K_{1}-1} & 0 & 0 \\
0 & v \boldsymbol{D}_{2, K_{2}-K_{1}+1} & 0 \\
0 & 0 & u \boldsymbol{D}_{2, M-K_{2}}
\end{array}\right] \text {. }
$$
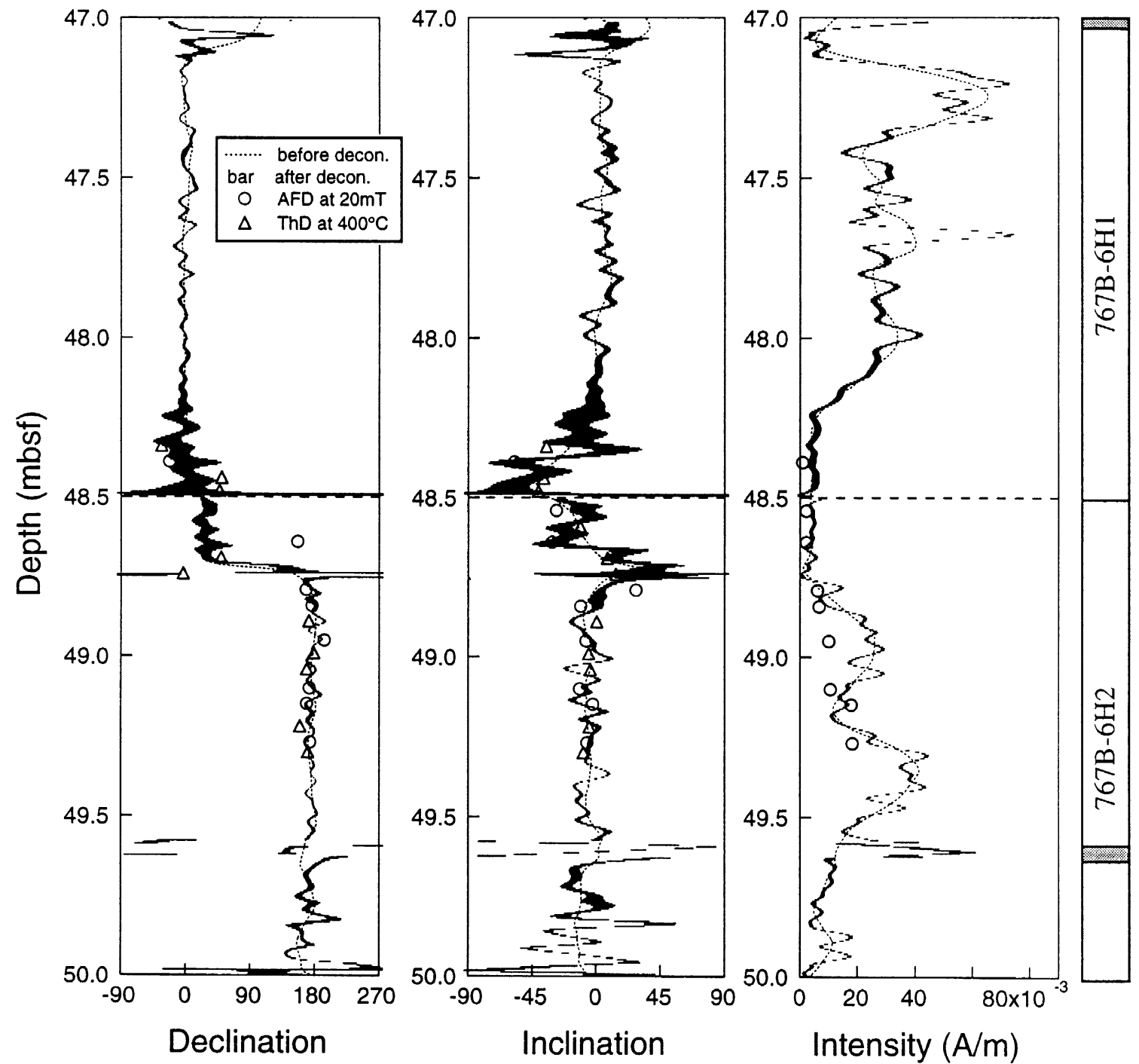

Fig. 2. The results of the modified ABIC-minimizing deconvolution. Captions are the same as in Fig. 1. Released data points are shown by shaded areas on the rightmost column along with the section names. Note the significant reduction in error-bar length compared with those in Fig. 1. See text for detailed explanation. 
Equation (13) is substituted in Eqs. (5b) and (9), and finally ABIC minimization is executed similarly to the case without the disturbed layer.

\section{Results}

The two advanced piston core (APC) samples of $1.5 \mathrm{~m}$ length from ODP Leg 124, Sections 767B-6H1 and 767B$6 \mathrm{H} 2$ were analyzed for the comparison between the previous single-parameter scheme and the new dual-parameter scheme. These core samples are composed of hemipelagic volcanogenic clayey silt and paleomagnetically stable because of strong magnetization intensity (Rangin et al., 1990). Paleomagnetic measurements were made at intervals of 5 $\mathrm{mm}$ after $20 \mathrm{mT}$ alternating field demagnetization (AFD) on board. In the raw data from Sections $767 \mathrm{~B}-6 \mathrm{H} 1$ and 2 there are no flux-jump miscounting or spike noise as reported for the pass-through data from Holes 769A and 769B by Oda and Shibuya (1996). Figure 1 shows the results by the deconvolution scheme by Oda and Shibuya (1996) which only one smoothness parameter applies to the two data sets separately. The magnetization obtained after the deconvolution are indicated by horizontal bars (95\% confidence limit) with the original magnetic moment before deconvolution (broken lines). Open circles and triangles correspond to the paleomagnetic results of discrete samples after AFD at $20 \mathrm{mT}$ and thermal demagnetization (ThD) at $400^{\circ} \mathrm{C}$, respectively. Note the large directional error at the polarity transition zone where magnetization intensity is low ( $48.5 \mathrm{mbsf})$.

Following the single-parameter deconvolution, the new dual-parameter scheme was applied to the same APC data sets. The magnetization at the top of Section 767B-6H1 were assumed to be seriously changing (lower smoothness) due to the obvious drilling disturbance (Rangin et al., 1990). This is also observable as disturbance in raw paleomagnetic data (Fig. 1). The first program which introduces two distinct layers was applied to Section 767B-6H1. As for Section 767B-6H2, magnetization shows strong intensity and large directional fluctuation at about 49.6 mbsf (Fig. 1) corresponding to the Australasian microtektite layer

Table 1. Results of ABIC-minimizing deconvolution.

\begin{tabular}{|c|c|c|c|c|c|c|c|c|}
\hline \multirow[t]{2}{*}{ Section } & \multirow{2}{*}{$\begin{array}{l}\text { Number of } \\
\text { layers }\end{array}$} & \multirow{2}{*}{$\begin{array}{c}\text { Number of } \\
\text { hyperparameters }\end{array}$} & \multicolumn{2}{|c|}{ Release } & \multirow{2}{*}{$\begin{array}{l}\text { Number of } \\
\text { released points }\end{array}$} & \multirow[t]{2}{*}{$\ln u$} & \multirow[t]{2}{*}{$\ln v$} & \multirow{2}{*}{$\begin{array}{l}\text { Minimum } \\
\text { ABIC }\end{array}$} \\
\hline & & & from & to & & & & \\
\hline \multirow[t]{7}{*}{ 767B-6H1 } & 1 & 1 & - & - & 0 & -1.5 & - & -19549 \\
\hline & 2 & 2 & 1 & 3 & 3 & -0.5 & -8.0 & -20566 \\
\hline & 2 & 2 & 1 & 5 & 5 & -0.5 & -7.5 & -20553 \\
\hline & 2 & 2 & 1 & 10 & 10 & -0.5 & -7.0 & -20508 \\
\hline & 2 & 2 & 1 & 15 & 15 & -0.5 & -7.0 & -20451 \\
\hline & 2 & 2 & 1 & 20 & 20 & -0.5 & -6.5 & -20405 \\
\hline & 2 & 2 & 1 & 30 & 30 & -0.5 & -6.5 & -20360 \\
\hline \multirow[t]{4}{*}{ 767B-6H2 } & 1 & 1 & - & - & 0 & -1.8 & - & -22216 \\
\hline & 3 & 2 & 216 & 225 & 10 & -1.0 & -3.5 & -22430 \\
\hline & 3 & 2 & 211 & 230 & 20 & -1.0 & -3.5 & -22395 \\
\hline & 3 & 2 & 201 & 240 & 40 & -1.0 & -3.0 & -22357 \\
\hline
\end{tabular}

(a)

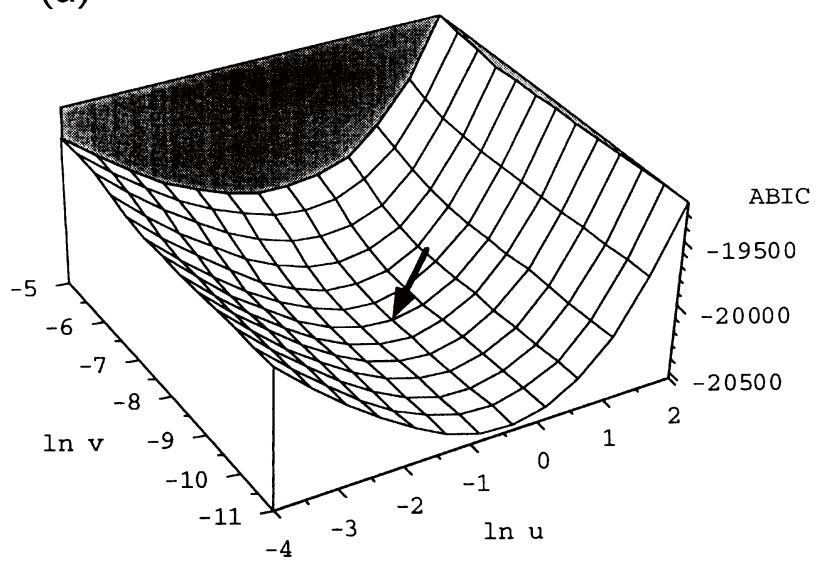

(b)

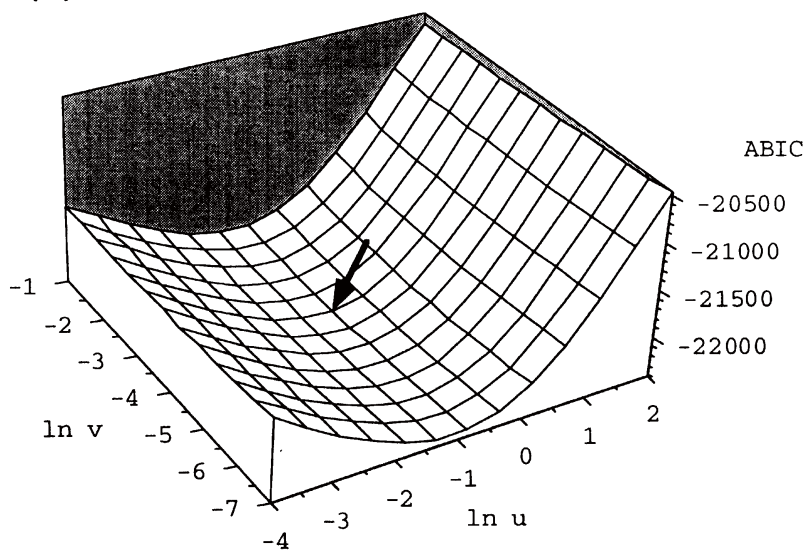

Fig. 3. (a) Bird's-eye view of ABIC versus $\ln u$ and $\ln v$ for 767B-6H1 assuming lower smoothness ( $v$ ) for data points between 1 and 3 . A stable minimum ABIC (arrow) is -20566 for $\ln u=-0.5$ and $\ln v=-8.0$. (b) The same plot for $767 \mathrm{~B}-6 \mathrm{H} 2$ assuming lower smoothness for data points from 216 to 225 . A stable minimum ABIC (arrow) is -22430 for $\ln u=-1.0$ and $\ln v=-3.5$. 
(Schneider et al., 1992). For this section, the second program assuming three distinct layers was applied. By releasing top 3 points $(0-1.5 \mathrm{~cm})$ for Section $767 \mathrm{~B}-6 \mathrm{H} 1$ and 10 points from 216 to $225(108-113 \mathrm{~cm})$ for Section 767B$6 \mathrm{H} 2$, we obtained better solutions which yielded a lower minimum ABIC compared with the former single-parameter model (Fig. 2). ABIC can be used for selecting the optimum model with respect to number of parameters and their values (Akaike, 1980). The intensity fluctuation and its error are quite large for the released short intervals, whereas the errors of the other parts are reduced by about a factor of 3 for 767B-6H1 and 1.5 for 767B-6H2.

Minimum ABIC was practically searched in the parameter space of $\ln v$ versus $\ln u$ on 0.5 interval grid points. The minimum ABIC values in the dual-parameter deconvolution are listed in Table 1 for each program with the results of the single-parameter deconvolution. For Section 767B-6H1, six cases were examined, in which top 3, 5, 10, 15, 20, and 30 data points are expressed by another parameter. ABIC comes to a minimum value of -20566 at $\ln u=-1.0$ and $\ln v$ $=-8.0$, when data points between 1 and 3 are released. ABIC values are plotted versus $\ln u$ and $\ln v$ in Fig. 3(a). This diagram shows a minimum in the trough as indicated by an arrow.

The Australasian microtektite layer, Section 767B-6H2 at about $110 \mathrm{~cm}$ (Schneider et al., 1992), was treated by a separate parameter released from the other part and the second program was used in its calculation. In the determination of released data points, we examined three different portions; 216-225, 211-230, and 201-240. The minimum $\mathrm{ABIC}$ is found to be -22430 for the partition from 216 to 225 . The magnetization intensity after deconvolution shows a sudden increase at $112 \mathrm{~cm}$ and a peak at $110 \mathrm{~cm}$. The number of microtektites counted per gram is 296 at $113 \mathrm{~cm}$, which abruptly reduces to 56 at $108 \mathrm{~cm}$ and 9 at $118 \mathrm{~cm}$ (Schneider et al., 1992). ABIC versus $\ln u$ and $\ln v$ are plotted for the optimum partition in Fig. 3(b). The minimum ABIC $(-22430)$ is detected at the point of $\ln u=-1.0$ and $\ln v=-3.5$
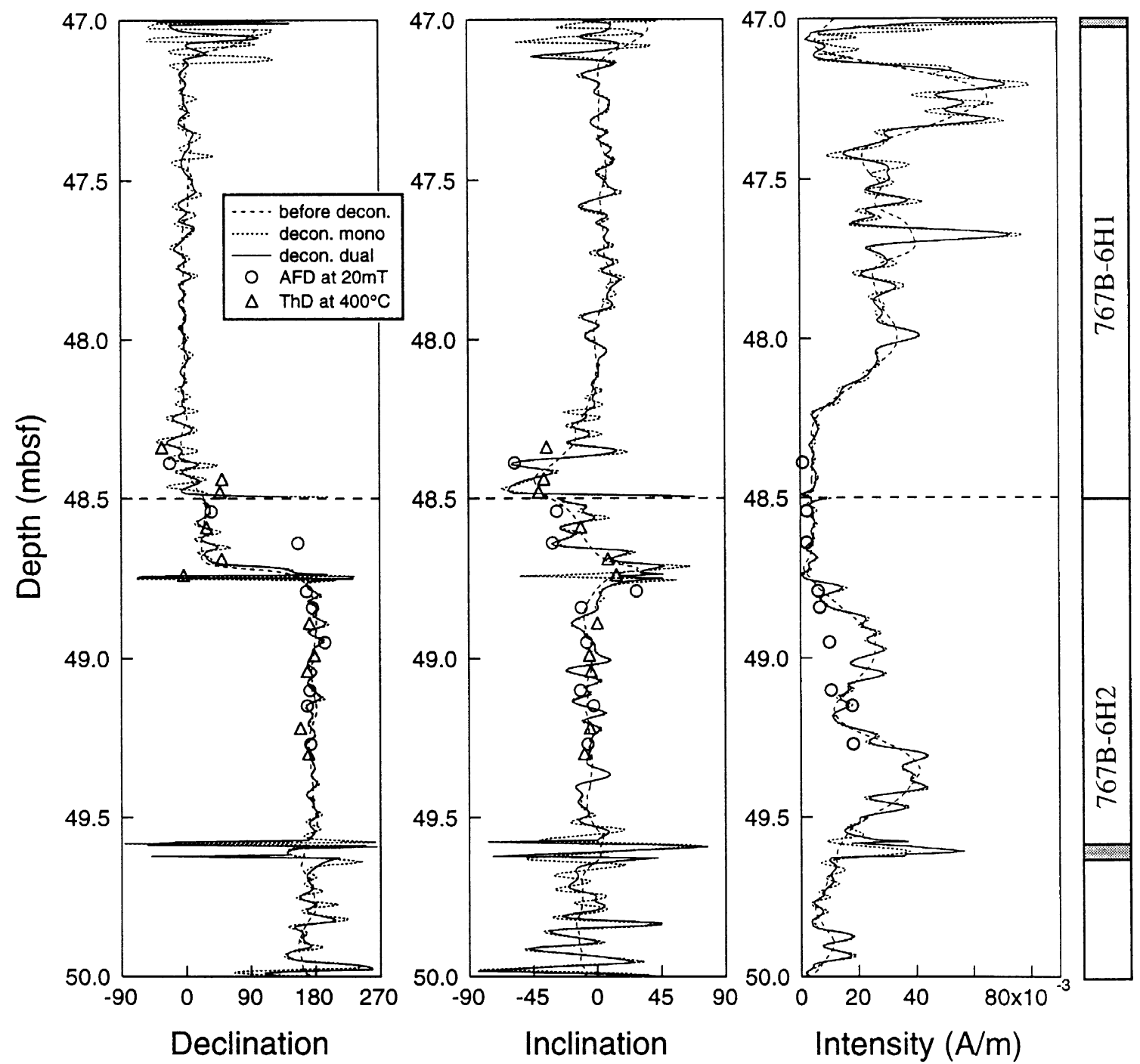

Fig. 4. The results of ABIC-minimizing deconvolution (Oda and Shibuya, 1996) for Section 767B-6H1 and 2 plotted with depth for declination, inclination and intensity. Solid (dotted) lines show magnetization after the deconvolution for the dual-parameter scheme (single-parameter). Broken lines indicate magnetization (raw data) before the deconvolution. Open circles and triangles represent paleomagnetic results from discrete cube samples after alternating field demagnetization at $20 \mathrm{mT}$ and thermal demagnetization at $400^{\circ} \mathrm{C}$, respectively. 
indicated by an arrow. The intensity peak may either be caused by a high concentration of magnetic minerals or magnetic inclusions in the microtektites.

\section{Discussion and Conclusions}

Figure 4 shows the results by the single-parameter scheme (dotted lines) and the dual-parameter scheme (solid lines) together with the raw magnetization before deconvolution (broken lines). The paleomagnetic results from discrete samples were also plotted by open circles (AFD at $20 \mathrm{mT}$ ) and open triangles $\left(\mathrm{ThD}\right.$ at $\left.400^{\circ} \mathrm{C}\right)$. Although the data points of the discrete samples seem to be closer to the magnetization after deconvolution than before, it is difficult to conclude which model follows the magnetization of discrete samples better. However, the modified scheme has advantages of the improvement both in minimum ABIC value and in the level of estimated errors. For Section 767B-6H1, intensity variations around 47.1-47.25 mbsf and directional fluctuations around 48.2-48.5 mbsf are clearly reduced by applying the dual-parameter scheme. For Section 767B-
$6 \mathrm{H} 2$, the magnetization fluctuation pattern above the microtektite layer is not significantly changed after the modification, whereas undulations around 49.63-49.80 mbsf are quite reduced. Figure 5 shows the same results in $x$-, $y$-, and $z$-components. As the $z$-component is small, all the characteristic features mentioned above can be seen in the $x$ and $y$-components, especially in the $y$-component.

In conclusion the modified ABIC-minimization deconvolution was successfully applied to the real passthrough measurement data from two APC samples of ODP. Minimum ABIC was reduced by assuming the second smoothness parameter for the disturbed core top of Section 767B-6H1 (two distinct layers), and the Australasian microtektite layer of Section 767B-6H2 (three distinct layers) where intensity of magnetization abruptly increases. The spurious fluctuations due to over-relaxation were reduced by the new dual-parameter deconvolution schemes. The errors were lowered by a factor of 3 for Section 767B-6H1 and 1.5 for Section 767B-6H2. These examples demonstrate that the present Bayesian model was significantly improved
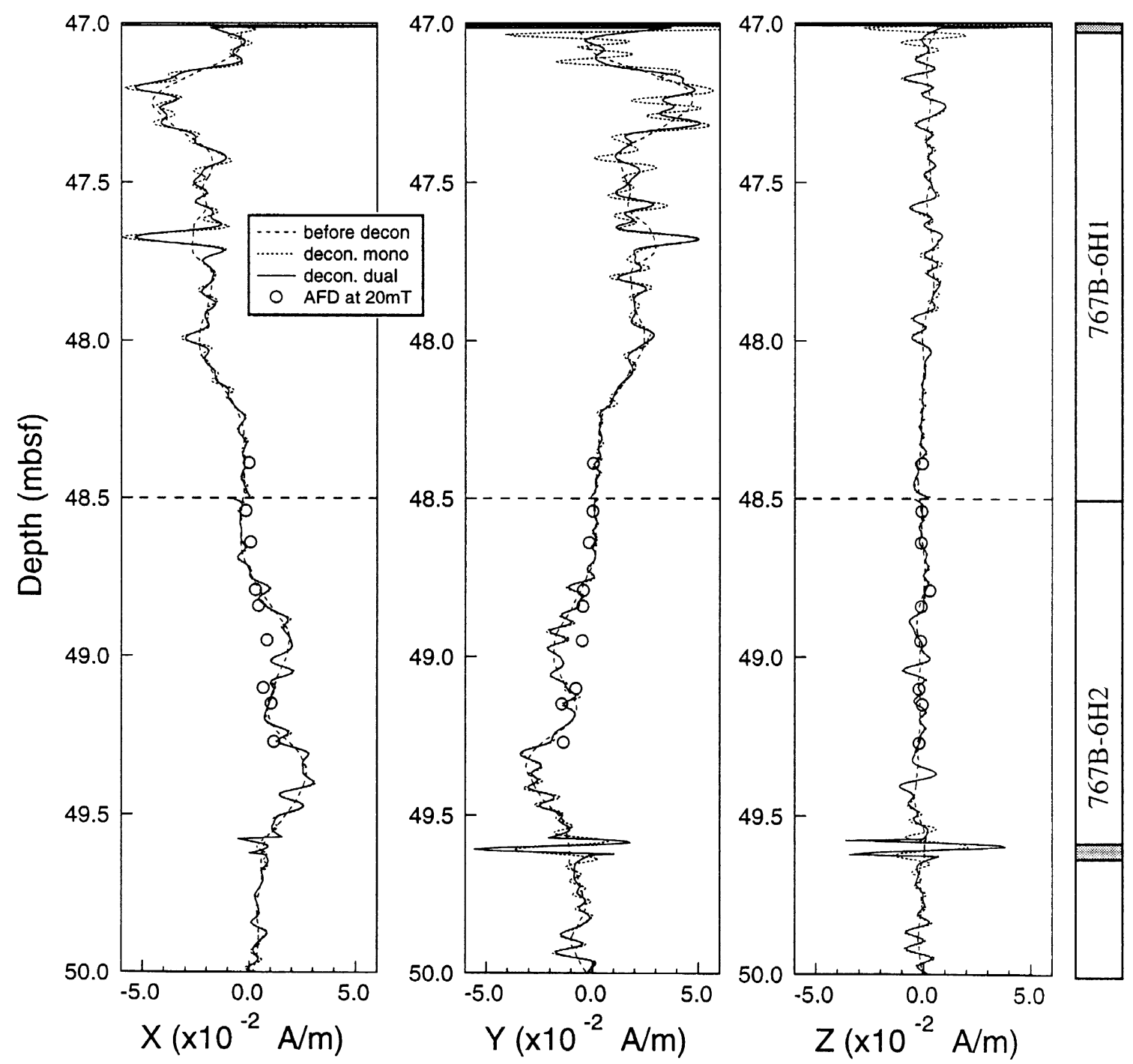

Fig. 5. The results of ABIC-minimizing deconvolution (Oda and Shibuya, 1996) for Section 767B-6H1 and 2, plotted with depth for $x$, $y$, and $z$ components of magnetization. Solid (dotted) lines show magnetization after the deconvolution for the dual-parameter scheme (single-parameter). Broken lines indicate magnetization before the deconvolution. Open circles represent paleomagnetic results of discrete cube samples after alternating field demagnetization at $20 \mathrm{mT}$. 
by introducing additional information, such as physical conditions of the samples other than magnetic remanence data itself, to the prior distribution of the data.

Acknowledgments. We are grateful to Drs. Dean Merrill and Vindell Hsü for their help on the pass-through measurements of the APC samples on-board. Great improvements were made on the manuscript according to the critical review by Dr. Hideo Thunakawa and an anonymous reviewer. We also thank Dr. Toshitsugu Yamazaki for his comments and Dr. Wietze van der Werff for his grammatical suggestions. The samples were supplied with the permission of the Ocean Drilling Program. The FORTRAN code of the deconvolution program is available upon request through e-mail at hoda@gsj.go.jp.

\section{References}

Akaike, H., Likelihood and the Bayes procedure, in Bayesian Statistics, edited by J. M. Bernardo, M. H. DeGroot, D. V. Lindley, and A. F. M. Smith, pp. 143-166, Univ. Press, Valencia, Spain, 1980.

Constable, C. and R. Parker, Deconvolution of long-core palaeomagnetic measurements—spline therapy for the linear problem, Geophys. J. Int., 104, 453-468, 1991.

Dodson, R., M. Fuller, and W. Pilant, On the measurement of the magnetism of long cores, Geophys. Res. Lett., 1, 185-188, 1974.

Oda, H., Detailed records of Brunhes/Matuyama geomagnetic polarity reversal, Ph.D. Thesis, Kyoto University, 1995.

Oda, H. and H. Shibuya, Deconvolution of whole-core magnetic remanence data by ABIC minimization, J. Geomag. Geoelectr., 46, 613-628, 1994

Oda, H. and H. Shibuya, Deconvolution of long-core paleomagnetic data of Ocean Drilling Program by Akaike's Bayesian Information Criterion minimization, J. Geophys. Res., 101, 2815-2834, 1996.

Rangin, C., E. A. Silver, M. T. von Breymann et al., Proc. ODP, Init. Repts. 124, 1990.

Schneider, D. A., D. V. Kent, and G. A. Mello, A detailed chronology of the Australasian impact event, the Brunhes-Matuyama geomagnetic polarity reversal, and global climate change, Earth Planet. Sci. Lett., 111, 395-405, 1992.

H. Oda (e-mail: hoda@gsj.go.jp) and H. Shibuya (e-mail: shibuya@sci.kumamoto-u.ac.jp) 